\title{
The influence of flexibility before strength and power exercise on jumps and sprints performance in gymnasts and dancers submitted to eight weeks of training
}

\author{
Isabella Righi, Arthur F. Gáspari, Ricardo Berton, Manoel P. da Silva, Junior Moriggi, Marco Carlos Uchida.
}

\begin{abstract}
The stretching exercises, flexibility ability, have a negative acute effect on maximal strength and power. For our knowledge, there aren't studies that confirm this interference chronically. The aim of this study was to verify the influence of flexibility training immediately before of power training, jumps and sprints, in adults who have practiced the Rhythmic gymnastics (GR), Artistic gymnastics (GA) and/or Dance (D) for at least 5 years. The participants were divided into two groups: Strength/Power group (GF/P) and Flexibility plus Strength/Power group (GF-F/P). Vertical jump and sprint tests were performed before, and after 8 weeks of intervention. The data were collected through of MyJump2 and MySprint apps. Both of the groups demonstrated similar increases on jump and sprint parameters (power), suggesting that there is not a negative interference on this ability, even when flexibility is applied before strength/power training.
\end{abstract}

E-mail: isabellarighi@terra.com.br

Key words: Stretching, Power, App.

\section{Introduction}

The physical abilities strength, power and flexibility are considered essentials ${ }^{2}$ for $\mathrm{GA}, \mathrm{GR}$ and $\mathrm{D}$. The training of these abilities is basic for performance of athletes, but, the best way to organize training sessions is not clear yet. According Bogdanis' et al. study, there is a negative impact in vertical jump after a static stretching exercise in gymnasts. So it is suggested that both training, flexibility before power one, has to be avoided for strength and power development. However it is not known what is the impact in longitudinal study. Therefore, the aim of this is to verify the effect of jumps preceded by stretching exercises in GA, GR, or D practitioners.

\section{Results and Discussion}

Fourteen participants ( 11 women and 3 men), at least five years of practice of the GA, GR and/or D, were enrolled in 8-week training duration (Figure 1), and they were separated in two groups: 1) Strength and Power $(G F / P)$; 2) Flexibility before Strength/Power (GF-F/P).

Training sessions occurred twice a week, lasting approximately one hour. Training were compose as following: GF/P - 5min warm up followed by three jumping exercises (counter movement vertical jump, drop jump and vertical jump); GF-F/P performed the same warm up followed by three stretching exercises (forced hip and knee flexion and hip extension) immediately before the same three jumps exercise of GF/P. Pre and Post data collection for jump and Sprint occurred by MyJump2 and MySprint apps, respectively.

Figure 1. Experimental design.

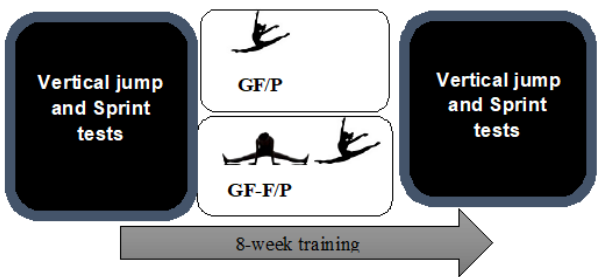

Scientific literature have been shown a negative acute influence in power performance, when stretching is applied before strength and/or power assessments. Interestingly, in our longitudinal study, it was verified that both of the groups increased the strength and power variables, without difference between groups, Table 1.

Table 1. Jump and Sprint.

\begin{tabular}{|c|c|c|c|c|c|c|}
\hline & \multicolumn{3}{|c|}{ GF/P (n. 7) } & \multicolumn{3}{|c|}{ GF-F/P (n. 7) } \\
\hline & Pre & Post & $\Delta(\%)$ & Pre & Post & $\Delta(\%)$ \\
\hline \multicolumn{7}{|l|}{ Jumps } \\
\hline $\mathrm{SJ}(\mathrm{cm})$ & $26.4 \pm 9.5$ & $29.8 \pm 8.8^{*}$ & $16 \pm 18.5$ & $29 \pm 7.1$ & $31.9 \pm 6.1^{*}$ & $11.8 \pm 14.5$ \\
\hline $\mathrm{CMJ}(\mathrm{cm})$ & $27.3 \pm 8.6$ & $31.3 \pm 9.7^{*}$ & $15 \pm 9.7$ & $29.3 \pm 7.5$ & $32.9 \pm 7.0^{*}$ & $14.6 \pm 21.3$ \\
\hline \multicolumn{7}{|l|}{ Sprint } \\
\hline $\begin{array}{l}\text { Time } 30 \mathrm{~m} \\
\text { (s) }\end{array}$ & $5.5 \pm 0.6$ & $5.5 \pm 0.6$ & $0.1 \pm 5.7$ & $5.3 \pm 0.3$ & $5.4 \pm 0.4$ & $0.4 \pm 3.0$ \\
\hline $\begin{array}{l}V \max \\
(\mathrm{m} / \mathrm{s})\end{array}$ & $6.5 \pm 1.1$ & $6.3 \pm 1.0^{*}$ & $-3.9 \pm 5.9$ & $6.8 \pm 0.4$ & $6.5 \pm 0.5^{*}$ & $-4.5 \pm 2.2$ \\
\hline FO (N/kg) & $8.8 \pm 1.0$ & $10.9 \pm 1.0^{*}$ & $25.1 \pm 15.9$ & $8.1 \pm 1.5$ & $9.2 \pm 1.2^{*}$ & $14.7 \pm 6.6$ \\
\hline $\begin{array}{l}\mathrm{P} \max \\
(\mathrm{W} / \mathrm{Kg})\end{array}$ & $14.8 \pm 3.5$ & $17.2 \pm 3.4^{*}$ & $18.9 \pm 20.8$ & $14.1 \pm 2.7$ & $17.8 \pm 8.2^{*}$ & $22.8 \pm 29.3$ \\
\hline
\end{tabular}

Data presented as mean \pm standard deviation. The values of the sprint and jump variables, pre and post intervention of both groups were compared through ANOVA of two-way for repeated measurements, with *indicating the main time effect. The level of significance adopted was $p<0,05$. SJ - Squat Jump; CMJ Counter Movement Jump; Time 30m - Total time for go through 30m; Vmax maximum speed on Sprint; FO - estimated vertical force; Pmax - estimated maximum power.

\section{Conclusions}

The flexibility training does not impact negatively in power training, even when they are trained in the same session. It suggests that this sequence, stretching and power training, is not harmful for gymnast and dancing practitioners who desire develop muscle power.

Acknowledgement

To participants, FEF-Unicamp and PRP-Unicamp. 